\title{
EVALUACIÓN DE LA ASTENIA EN PACIENTES ONCOLÓGICOS AVANZADOS Y UNA PROPUESTA DE PROTOCOLO DE INTERVENCIÓN PSICOLÓGICA
}

\author{
FATIGUE ASSESSMENT IN ADVANCED CANCER PATIENTS. A PROPOSAL OF \\ PSYCHOLOGICAL INTERVENTION GUIDELINE FOR MANAGEMENT FATIGUE
}

\begin{abstract}
Laura María Haro', Carolina Ortega ${ }^{2}$, María Teresa de las Heras ${ }^{3}$, Ángeles Poyatos 4 ,
\end{abstract} María José Molina ${ }^{2}$, María del Mar Muñoz ${ }^{2}$, José Antonio Santiago ${ }^{2}$ y Amaya Olaverri

\author{
Unidad de Psicooncología. AECC. Hospital Virgen de la Luz. Cuenca \\ 2 Servicio de Oncología Médica. Hospital Virgen de la Luz. Cuenca \\ 3 Atención Primaria del Área de Cuenca \\ 4 Unidad de Cuidados Paliativos. Hospital Virgen de la Luz. Cuenca
}

Resumen

La astenia es un síntoma con una elevada incidencia en pacientes con cáncer. Es un fenómeno multifactorial que deteriora la calidad de vida del paciente, con repercusiones físicas, psicológicas, sociales y laborales. Es uno de los síntomas que más preocupan al enfermo, incluso más que el dolor, y al que se le presta poca atención. Por tanto se infravalora su importancia y no se aplican los medios diagnósticos necesarios para identificar sus causas y poder disminuirlas en la medida de lo posible.

El objetivo de este artículo es evaluar la percepción subjetiva de astenia en pacientes oncológicos avanzados, el malestar subjetivo que les produce la situación que están viviendo y valorar si utilizan estrategias para minimizar la sensación de cansancio. Consideramos necesario establecer un consenso sobre los instrumentos de medida a utilizar para poder comparar resultados.

Asimismo, se expone un protocolo de intervención psicológica para combatir la astenia fomentando la adaptación y el ajuste del paciente oncológico avanzado a su estado para aumentar con ello la sensación de bienestar y su calidad de vida.

Palabras clave: Astenia, fatiga, calidad de vida, cáncer avanzado, malestar subjetivo, intervención psicológica.
Abstract

Fatigue is a symptom with a high incidence in patients with cancer. It is a multifactorial phenomenon, which deteriorates the quality of life of patients, from physical, psychological, social and working points of view. It is one of the symptoms that most concern the patient, even more than pain, medically it is paid little attention, therefore underestimating its importance and not applying the diagnostic tools necessary to identify its causes and to reduce it as far as possible.

The aim of this paper is to assess the subjective perception of fatigue in advanced cancer patients, the subjective distress that produces the situation they are living and to assess whether they use strategies to minimize the feeling of tiredness. We consider it necessary to establish a consensus on the measuring instruments used to compare results.

We have developed a psychological intervention guideline for management fatigue by promoting the adaptation and adjustment of advanced cancer patients to illness and therefore increase sense of well being and quality of life.

Key words: Asthenia, fatigue, quality of life, advanced cancer, subjective distress, psychological intervention.

Correspondencia:

Laura María Haro Martínez

Asociación Española contra el Cáncer de Cuenca. Calle Las Encinas $364^{\circ}$ C. 16002 Cuenca

E-mail: laura.haro@aecc.es 


\section{Introducción}

La astenia es el síntoma más frecuente asociado a enfermos con cáncer avanzado y con sus tratamientos ${ }^{(1)}$. La 22. ${ }^{a}$ edición del Diccionario de la Lengua Española define la astenia (del griego astheniea) como «falta o decaimiento de fuerzas caracterizado por apatía, fatiga física o ausencia de iniciativa» ${ }^{(2)}$. En el ámbito clínico, la astenia tumoral se considera como la "sensación inespecífica y subjetiva de cansancio sin necesidad de esfuerzo previo, que repercute de manera significativa en el funcionamiento físico, psicológico o social del paciente, y que está asociada al diagnóstico de la enfermedad neoplásica. Es un cansancio que no se alivia con el reposo $^{(3)}$. El término astenia hace referencia a dos tipos de síntomas. El primero de ellos es la fatigabilidad fácil con disminución de la capacidad para mantener una actividad normal. El segundo sería una sensación anticipatoria de dificultad para iniciar determinadas actividades ${ }^{(4)}$. Es decir, la astenia es una debilidad sistémica con manifestaciones, tanto físicas como mentales. En lo somático se incluyen debilidad generalizada, disminución de la fuerza y mayor fatigabilidad, mientras que psicológicamente hay disminución de la capacidad para actividades intelectuales, labilidad emocional y apatía(5).

Para Cella, Davis y Breitbart la prevalencia de la astenia varía entre el 60-90\% de pacientes, dependiendo de los criterios diagnósticos utilizados (nivel de evidencia IV) ${ }^{(6)}$.

La verdadera incidencia de la astenia tumoral es difícil de precisar. Los estudios publicados estiman que afecta al $75 \%$ de los pacientes con cáncer avanzado o con metástasis óseas ${ }^{(7)}$.

Según Bruera, los factores que contribuyen a la astenia en pacientes con cáncer serían fundamentalmente: el dolor y la toxicidad de los fármacos, la caquexia, los tratamientos de quimioterapia (depende de la intensidad del tratamiento, número de ciclos y de los días transcurridos desde su administración) y radioterapia (relacionada con el volumen irradiado y el órgano incluido), las infecciones, las alteraciones metabólicas, el malestar psicólogico, la liberación de citoquinas, las alteraciones musculares, el fallo autonómico y la ane$\mathrm{mia}^{(8)}$. En este último caso, la presencia de anemia relacionada con el cáncer o con los tratamientos, sabemos hoy en día que es un factor que interviene de forma directa en la producción de astenia e incide de forma directa en la calidad de vida de los pacientes con cáncer e incluso en la respuesta al tratamiento y en la supervivencia. La anemia está asociada a un peor pronóstico y estado funcional. El impacto de la anemia en el performance status (PS) está respaldado por múltiples estudios. En ellos la baja concentración de hemoglobina $(<12 \mathrm{~g} / \mathrm{dl})$ se correlacionó con bajo $\mathrm{PS}^{(9)}$. La anemia severa (hemoglobina $<8$ $\mathrm{g} / \mathrm{dl}$ ) es causa conocida de astenia, aunque cifras menores de $12 \mathrm{~g} / \mathrm{dl}$ también pueden causarla ${ }^{(10)}$.

En cuanto a los trastornos psicológicos, la causa más frecuente de astenia en la población sin cáncer es un trastorno psiquiátrico: entre el 40 y el $60 \%$ de los pacientes que acuden a Atención Primaria por cansancio presentan en realidad un trastorno de ansiedad o depresión. Algunos estudios han encontrado una prevalencia de depresión en pacientes oncológicos que oscila entre el 15 y el $25 \%{ }^{(11)}$. Hay un trabajo realizado en mujeres con cáncer de mama avanzado en el que se intentó relacionar la astenia con diversas variables; de todas ellas sólo se encontró correlación estadísticamente significativa con la depresión ${ }^{(12)}$. En otro estudio se considera que la astenia sería una forma más de respuesta asociada al estrés ${ }^{(13)}$.

Si atendemos a la Guía de Práctica Clínica Oncológica, los criterios diagnósticos 
para la astenia relacionada con el cáncer son los siguientes ${ }^{(14)}$ : Tabla 1.

Aunque la aplicación de estos criterios permite realizar un diagnóstico preciso en la práctica diaria, habitualmente no se utilizan. Por tanto, son útiles en el campo de la investigación pero su sensibilidad es baja por lo que su aplicación estricta no permite diagnosticar de astenia tumoral a muchos pacientes con cáncer que se quejan de astenia.

Las herramientas para evaluar la astenia deben medir la intensidad, temporalidad, factores que la exacerban y alivian, así como su impacto sobre la calidad de vida. Los métodos de evaluación más utilizadas pueden agruparse en cuatro grupos: test de capacidad funcional (Treadmill performance), evaluación subjetiva de la capacidad funcional (Listado de Pearson y Byars de percepción de astenia), estimadores de funcionalidad objetiva (ECOG, EFAT), y evaluación subjetiva de la astenia (EVA, FACT o BFI). A su vez las escalas pueden ser unidimensionales o multidimensionales. Las últimas son más difíciles de aplicar y su empleo está limitado(15).

La evidencia sobre la eficacia de la terapia farmacológica es escasa y constituye un área que requiere más investigación. Existen tres ensayos aleatorizados que han mostrado que los corticoides disminuyen la astenia, aunque el mecanismo de acción es desconocido (nivel de evidencia (b) $)^{(16)}$. Sin embargo, su uso continuado puede contribuir a la astenia induciendo miopatía, infección y otras complicaciones. Otros ensayos controlados randomizados han mostrado que psicoestimulantes como el metilfenidato y la dextroanfeta-

\section{Tabla 1. Criterios diagnósticos para la astenia relacionada con el cáncer ${ }^{(1)}$}

A. Los siguientes síntomas se han presentado cada día o casi cada día, durante un periodo de dos semanas en el último mes:

- Astenia significativa, disminución de energía o aumento desproporcionado de la necesidad de descansar y sin cambios en el nivel de actividad.

- Y por lo menos cinco de los siguientes:

- Quejas de debilidad generalizada o pesadez de miembros.

- Disminución de concentración o atención.

- Disminución de la motivación o interés por las actividades cotidianas.

- Insomnio o hipersomnia.

- Experiencia de sueño no reparador.

- Percepción de la necesidad de luchar para superar la inactividad.

- Reactividad emocional marcada (tristeza, frustración o irritabilidad) a la sensación de fatiga.

- Dificultad para completar las tareas diarias atribuidas a la sensación de fatiga.

- Percepción de problemas con la memoria a corto plazo.

- Malestar tras el esfuerzo que dura varias horas.

B. Los síntomas causan distrés significativo a nivel clínico, social, ocupacional o en otras áreas funcionales importantes.

C. Hay evidencias por la historia, examen físico o datos de laboratorio de que los síntomas son consecuencia del cáncer o del tratamiento del cáncer.

D. Los síntomas no son consecuencia de trastornos psiquiátricos asociados como depresión mayor, trastornos de somatización o delirio. Nivel de evidencia IV. 
mina pueden antagonizar los efectos sedantes de los opioides (nivel de evidencia Ib) pero no hay evidencia de que mejoren la astenia que no se debe a toxicidad por opioides $^{(17)}$. Futuras posibilidades de tratamiento incluirían agentes que prevengan la liberación de citoquinas o inhibidores de la acción de citoquinas ${ }^{(18)}$.

En cuanto al tratamiento de la anemia, el North Central Cancer Treatment Group (NCCTG) inició su primer estudio para mejorar la anemia relacionada con la quimioterapia, demostrando que la eritropoyetina (40.000Ul U/semanal) era mejor que el placebo para aumentar los niveles de hemoglobina y disminuir las transfusiones en pacientes con anemia ${ }^{(19)}$.

Las medidas no farmacológicas incluyen educación e intervenciones psicosociales que han mostrado ser útiles en la astenia. La educación y el counselling sobre la astenia antes de empezar el tratamiento antineoplásico, durante y después, pueden ayudar a reducir el estrés. Se recomienda dar consejos sobre estrategias para tolerar mejor la astenia y adaptarse a la nueva situación ${ }^{(20)}$.

Las intervenciones sobre el estrés, la depresión y la ansiedad ayudan a reducir la astenia y a mejorar el humor. Se utilizan fundamentalmente terapias cognitivas, técnicas de relajación e hipnosis o intervenciones conductuales con técnicas de adaptación $^{(21,22)}$.

Diversos estudios sugieren que los programas de ejercicios físicos pueden mejorar la capacidad funcional, aumentar la masa muscular, la ventilación pulmonar, reducir el esfuerzo necesario para realizar las actividades diarias y disminuir la sensación de astenia. El ejercicio físico es la intervención que tiene mayor evidencia de beneficio terapéutico ya que mejora el humor y la calidad del sueño ${ }^{(23)}$.

El objetivo primordial de nuestro trabajo es conocer el punto de vista que el enfermo oncológico tiene acerca del su- ceso vital estresante que está sufriendo y que englobaría el afrontamiento de sus últimos días, la valoración subjetiva de la sensación de cansancio (como uno de los síntomas fundamentales presentes en este momento) y la capacidad para usar alguna técnica que le permita aliviar dicho síntoma.

Con este propósito hemos diseñado un estudio donde recogemos estos datos mediante una evaluación que detallamos a continuación.

\section{MÉTODO}

\section{Sujetos}

La muestra está formada por 25 pacientes (10 mujeres y 15 hombres) diagnosticados de enfermedad oncológica avanzada (con diferentes tipos de cáncer) donde el tratamiento es fundamentalmente paliativo. La media de edad de los pacientes estudiados se sitúa en torno a los 68,76 años. Los criterios de inclusión fueron los siguientes: ausencia de disfunciones cognitivas y consentimiento verbal por parte del paciente para participar en el estudio.

\section{Evaluación y Procedimiento}

Son evaluados cuando acuden a la consulta externa de Oncología Médica del Hospital Virgen de la Luz de Cuenca durante el mes de marzo de 2010. Por tanto se trata de un estudio transversal de 15 días de duración. La entrevista se realizaba de manera individual por el psicólogo.

No existe un estándar universalmente aceptado para evaluar la astenia; por tanto, es evidente la dificultad para medir este síndrome complejo y subjetivo.

En nuestro estudio tuvimos en cuenta tres factores relacionados con la aparición de astenia:

1.- Si el paciente estaba recibiendo tratamiento con quimioterapia paliativa. 
2.- La aparición de malestar psicológico a través de la medición del nivel de ansiedad.

3.- Presencia o ausencia de anemia.

A través de la anamnesis se recogieron aspectos relacionados con la aparición de la astenia como características clínicas de la misma, evaluación de factores predisponentes y etiológicos y de la calidad de vida.

Para ello utilizamos la escala visual analógica (EVA) desarrollada por el grupo Asthenos que está basada en la escala LASA (Linear Analog Scale Assessment) por su brevedad y su facilidad de aplicación. Es un instrumento validado para la detección y el tratamiento de la astenia y la anemia (ver tabla 2). Está formada por tres preguntas:

1.- ¿Cómo evaluaría usted su nivel de energía durante la semana pasada?
2.- ¿Cómo evaluaría usted su nivel de habilidad para realizar sus actividades diarias durante la semana pasada?

3.- ¿Cómo evaluaría su calidad de vida general durante la semana pasada?

Por otro lado hemos usado un termómetro de malestar para conocer el nivel de ansiedad ante esta difícil situación. Los pacientes otorgan una puntuación de 0-100 USAS (Unidad Subjetiva de Ansiedad), según se expone en la tabla 3 (ver tabla 3):

\section{RESULTADOS}

Se trata de un estudio descriptivo. Los resultados se obtuvieron del total de pacientes que componían la muestra $(\mathrm{n}=25)$.

Las variables que se consideraron fueron la edad, el tratamiento con quimioterapia, la presencia de anemia, el marcador

\section{Tabla 2. Escala visual analógica (EVA) desarrollada por el grupo Asthenos}

Nivel de energía durante la última semana

0. 10

El menor posible

El mayor posible

Capacidad para realizar las actividades de la vida diaria durante la última semana

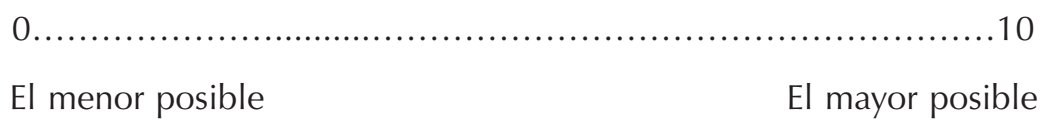

Calidad de vida global durante la última semana 0. 10

El menor posible

El mayor posible 
Tabla 3. Termómetro del malestar

\begin{tabular}{|l|l|}
\hline 0 & Me siento tranquilo \\
\hline 20 & Me encuentro algo intranquilo \\
\hline 40 & Me siento intranquilo \\
\hline 60 & Me noto bastante intranquilo \\
\hline 80 & $\begin{array}{l}\text { Me siento muy intranquilo y } \\
\text { tenso }\end{array}$ \\
\hline 100 & $\begin{array}{l}\text { Esta situación me hace sentir tan } \\
\text { ansioso y tenso que no puedo } \\
\text { resistirlo }\end{array}$ \\
\hline
\end{tabular}

de ansiedad, la utilización de estrategias y la calidad de vida.

La media de edad está en 68,76 años incluyendo hombres y mujeres.

El 56\% de ellos estaban recibiendo, en el momento en el que son evaluados, tratamiento con quimioterapia paliativa mientras que el $44 \%$ sólo recibían tratamiento sintomático.

$\mathrm{Si}$ atendemos a la posible relación entre los factores astenia y anemia podemos observar que sólo el $25 \%$ de los pacientes del estudio presentan niveles anormales de hemoglobina. Consideramos que existe anemia, de acuerdo con los criterios de la OMS, cuando los valores de hemoglobina son inferiores a $13 \mathrm{~g} / \mathrm{dl}$ en varones y menores de $12 \mathrm{~g} / \mathrm{dl}$ en mujeres. De los pacientes que presentaban anemia, el $66,6 \%$ eran varones y el $33,4 \%$ mujeres.

En cuanto a la percepción subjetiva de astenia, valorada mediante la escala visual analógica, hemos hallado que el $64 \%$ de los pacientes puntúan su nivel de energía durante la última semana por encima de 5. La puntuación puede variar entre el valor 0 (gran cansancio, nada de energía) hasta 10 (ausencia de cansancio, no se ha sentido cansado debido a su estado de salud). Con respecto a la percepción de la capacidad para realizar sus habilidades de la vida diaria, obtenemos que el $56 \%$ de ellos afirma que, durante la semana anterior, pudieron realizar de forma adecuada dichas actividades otorgando puntuaciones en la escala por encima de 5. El 44\% restante de los pacientes tuvieron algunas dificultades para realizar actividades de la vida diaria. Por último, más de la mitad de los pacientes (56\%) consideran bastante aceptable su calidad de vida dándole puntuaciones superiores a 5 .

Si nos centramos en el malestar subjetivo que les produce la situación que están viviendo, el $47,38 \%$ hablan de un malestar intenso (puntuación $>80$ ), el $21,05 \%$ presentan un malestar intermedio (entre $40 \mathrm{y}$ 60) y sólo un $31,57 \%$ informan de un bajo malestar (puntuaciones inferiores a 40).

Otro factor analizado se refiere a la utilización de estrategias para disminuir la sensación de cansancio. La mitad de ellos, aproximadamente el 52\%, informan de que realizan actividades de ocio que les ayudan a ocupar el tiempo. Destacan fundamentalmente actividades como un ejercicio físico moderado (caminar alrededor de 30 minutos al día), dormir, leer el periódico, escuchar la radio, ver la televisión, hacer pasatiempos (crucigramas, sopas de letras), ir a ver a los amigos, practicar yoga, ordenar papeles, charlar con conocidos y familiares.

\section{PROTOCOLO DE INTERVENCIÓN PSICOLÓGICA EN PACIENTES CON CÁNCER AVANZADO}

Consideramos la intervención psicológica como una pieza fundamental para combatir la astenia. Por ello hemos elaborado un protocolo basado en técnicas sencillas que tienen como finalidad mejorar la adaptación del enfermo a su estado y conseguir disminuir la sensación de malestar.

Resulta de gran interés poder hacer una correcta prevención de un síntoma que llega a afectar a más del $90 \%$ de los pacientes con cáncer en cualquier 
momento de la evolución de su enfermedad, pero dada la complejidad por la que aparece este síntoma, su control y su prevención no parecen nada sencillos. Es por ello que parece obvio, que la profilaxis debe orientarse hacia el control de síntomas y el apoyo emocional de los pacientes con cáncer.

Lo adecuado es anticiparse a la aparición de síntomas que suelen acompañar a la enfermedad en fase avanzada o síntomas derivados de la utilización de tratamientos quimioterápicos y radioterápicos. Sería por tanto de interés establecer pacientes de riesgo en los que se pueda prevenir o corregir diversas alteraciones que puedan conducir al desarrollo de astenia $^{(24)}$. No debemos de olvidar el papel de la adecuada valoración psicológica y apoyo psicológico en aquellos pacientes que presentan riesgo o cierto grado de distrés emocional.

Los factores psicológicos por tanto tienen una incidencia directa en la presencia de la astenia en el paciente con cáncer avanzado; de hecho, la astenia puede ser aliviada con actividades que promuevan el descanso y la recuperación para que el paciente pueda adaptarse a su nuevo estado consiguiendo con ello un mayor grado de bienestar ${ }^{(25)}$.

La intervención psicológica va a tener como objetivos:

1) Aliviar el síntoma.

2) Aumentar el control sobre la situación.

3) Normalizar el estado emocional del paciente con astenia.

Las técnicas psicológicas seleccionadas están basadas en las estrategias propuestas por Lacasta et. al. ${ }^{(25)}$.

- Psicoeducación sobre la astenia para disminuir la incertidumbre $y$ aumentar la participación del paciente. El objetivo es conocer mejor el síndrome de astenia crónica aso- ciada al cáncer para que el paciente ponga en marcha una serie de intervenciones eficaces que le permitan mejorar su calidad de vida.

- Técnicas de reestructuración cognitiva que pretenden identificar $y$ trabajar creencias erróneas o pensamientos distorsionados con respecto al cansancio. La finalidad es que el enfermo afronte su situación con un espíritu de lucha; colaborando en el tratamiento médico y mejorando su percepción de control.

- Favorecer la "ventilación emocional" enseñando al paciente a expresar sus sentimientos y ayudándoles a validar sus emociones de tristeza, angustia, rabia, ira o irritabilidad.

- Fomentar la realización de actividades. Puede ser interesante que el paciente apunte en un diario motivaciones y objetivos realistas. Es aconsejable que los enfermos deleguen o pospongan aquellas actividades que no sean imprescindibles. Deben realizarlas en aquellos momentos del día con menor cansancio y aprovechar para descansar y relajarse cuando se perciba mayor sensación de agotamiento. Es importante el autocuidado personal porque va a aumentar su nivel de autoestima y a mejorar la sensación de bienestar. Incluir actividades de ocio y tiempo libre que favorezcan la distracción de la atención: los juegos, la música, la lectura y la socialización disminuyen la astenia.

- Proponer un programa de ejercicio físico moderado. El ejercicio reduce en un $40-50 \%$ los niveles de astenia. Puede ser suficiente caminar un rato cada día. En aquellos pacientes con tumores avanzados en fase terminal, parece de utilidad el uso de fisioterapia y terapia ocupacional(26). A la hora de planificar un progra- 
ma de ejercicios deben tenerse en cuenta las características propias de los pacientes como edad, comorbilidad, tipo de cáncer o capacidad física previa. Hay que descartar la presencia de condiciones que lo contraindiquen como metástasis óseas.

- Terapia de recuperación de la atención. La focalización permanente de la atención en la sensación de cansancio puede incrementarla. Se sugiere dirigir la atención a otros estímulos diferentes. Actividades como sentarse en el parque y contemplar el paisaje pueden ayudarnos a relajarnos y centrarnos en otras cosas.

- Establecer una buena higiene de sueño. Las alteraciones del sueño en el enfermo oncológico oscilan desde el insomnio hasta la hipersomnia. Aunque el paciente neoplásico dedica más tiempo a descansar, el sueño tiene peor calidad y las interrupciones son frecuentes ${ }^{(27)}$. Por lo tanto, mantener un ritmo sueño-vigilia lo más regular posible; reducir progresivamente la actividad antes de acostarse; establecer rutinas antes de ir a dormir (tomar una infusión o baño relajante antes de acostarnos pueden ayudarnos a descansar); ir a la cama sólo cuando se sienta sueño; utilizar la cama sólo para dormir y no realizar otras actividades distractoras como leer, ver la televisión o escuchar música; evitar tomar excitantes como cafeína, tabaco o alcohol; eliminar estímulos molestos de la habitación y mantener un entorno silencioso y confortable.

- Entrenamiento en relajación muscular progresiva para normalizar el estado emocional reactivo al cansancio, mejorar la percepción subjetiva de astenia y potenciar el sentimiento de autocontrol del paciente, disminuir la respuesta de estrés y aliviar el sufrimiento ${ }^{(28)}$. Algún estudio sugiere que los programas con técnicas de relajación mejoran la calidad del sueño y la actividad durante el día ${ }^{(29,30)}$.

\section{DISCUSIÓN/CONCLUSIONES}

La astenia es un síndrome multidimensional difícilmente evaluable por los diversos aspectos que la conforman. En este estudio se han tenido en cuenta varios factores clínicos y psicológicos pero, para una evaluación completa, deberíamos incluir más síntomas no tenidos en cuenta. Sin embargo es complicado mantener el equilibrio, es decir, realizar una evaluación exhaustiva pero que no sea demasiado prolija, de forma que no agote al paciente. Nosotros pudimos observar que los pacientes no encontraban dificultad para responder a las preguntas formuladas ya que el tiempo de aplicación era breve (2-3 minutos). No se mostraban cansados al final del ejercicio.

Los pacientes consideran que la astenia es un síntoma presente e importante en su enfermedad. Aproximadamente el $48 \%$ de los entrevistados se quejaban de estar más cansados sin haber realizado un esfuerzo excesivo lo que en ocasiones limitaba el que pudieran realizar las actividades de la vida diaria al mismo ritmo que antes. Es decir presentaban alguna dificultad como necesitar más tiempo o tener que adaptarse a un horario en el que estaban más descansados aunque más de la mitad no habían dejado de realizar estas actividades.

La astenia es el efecto secundario más frecuente de la quimioterapia y la radioterapia. Se ha sugerido que puede estar relacionada con la presencia de anemia y el aumento de productos de desecho celulares secundarios a la destrucción ce- 
lular inducida por estos tratamientos. Más de la mitad de los pacientes estaban recibiendo tratamiento quimioterápico lo que puede contribuir a aumentar la sensación de cansancio.

La anemia parece que no justifica la sensación subjetiva de cansancio que presentan los pacientes ya que sólo el 25\% presentaban niveles anormales. Al tratarse de una causa reversible se Ilevaron a cabo las intervenciones indicadas (ferroterapia, ácido fólico, vitamina B12, transfusión de sangre o administración de eritropoyetina). Sin embargo, aunque los niveles de anemia mejoraron objetivamente, los pacientes seguían informando de la sensación de agotamiento y debilidad.

Por otro lado, encontramos que la astenia podría estar asociada con síntomas psicológicos como la ansiedad. Los pacientes presentaban niveles patológicos de estrés. Aunque no evaluamos su estado de ánimo de forma objetiva, la mayor parte de ellos afirmaban sentirse tristes y abatidos por la situación que estaban atravesando. A pesar de todo, el 56\% consideraban aceptable (puntuaciones $>5$ ) su calidad de vida y se esforzaban por llevar a cabo estrategias para disminuir el cansancio.

Al terminar la evaluación, entregamos una guía adaptada para ellos (basada en el protocolo arriba indicado) donde aparecían consejos sencillos para favorecer la adaptación a la astenia. Podemos resaltar que la mayoría aceptaron con buen agrado las pautas.

Sería conveniente seguir recogiendo información para completar el estudio. Deberíamos ampliar la muestra ya que consideramos que es insuficiente para sacar conclusiones definitivas y además sería interesante evaluar si los pacientes han llevado a cabo el programa de actividades propuesto para ver si ha mejorado su nivel de astenia y la valoración en su calidad de vida.

\section{REFERENCIAS BIBLIOGRÁFICAS}

1. Sanmartín J. Guías clínicas de Cuidados Paliativos. Arán; 2007. p.117.

2. Real Academia Española. Diccionario de la Lengua Española, 22. a edición. Espasa Calpe; 2001. p. 233.

3. Lacasta MA, Calvo C, González Barón M, Ordónez, A. Aspectos psicológicos de la astenia. Psicooncología 2004; 1 (2-3): 29-44.

4. Gándara I, Feliu J, Casado E. Complicaciones metabólicas del enfermo con cáncer. En: González Barón M, Ordóñez A, Feliu J, Zamora P, Espinosa E. Tratado de medicina paliativa y tratamiento de soporte en el enfermo con cáncer. Panamericana; 1996. p. 540-57.

5. Theologides A. Asthenia in cancer. Am J Med 1982; 73: 1-3.

6. Cella D, Davis K, Breitbart W, Curt G. Fatigue Coalition. Cancer-related fatigue: prevalence of proposed diagnostic criteria in a United States sample of cancer survivors. J Clin Oncol 2001; 19: 3385-91. Doi: 0732-183X/01/1914-3385

7. Feliu J, Belda-Iniesta C. Tratamiento de la astenia. En: González Barón M, Ordóñez A, Feliu J, Zamora P, Espinosa E, editores. Tratado de medicina paliativa y tratamiento de soporte en el enfermo con cáncer. Panamericana; 2007. p. 443-52.

8. Bruera E, Sweeney C. Cachexia and astenia in cancer patients. Lancet Oncol 2000; 1: 138-46. Doi:10.1016/S14702045(00)00033-4.

9. Ludwig H, Van Belle $S$, Barrett-Lee P, Birgegard G, Bokemeyer C, Gascón P, et al. The European Cancer Anemia Survery: A large multinational prospective survey defining the prevalence, incidence and treatment of anemia in cancer patients. Eur J Cancer 2004; 40: 2293-2306. Doi:10.1016/j. ejca.2004.06.019.

10. Barnes EA, Bruera E. Fatigue in patients with advanced cancer. A review. Inter J Gynecol Cancer 2002; 12: 424-8. Doi: 10.1046/ j.1525-1438.2002.t01-1-01147.x. 
11. Henriksson MM, Isometsa ET, Hietanen PS, Aro HM, Lonnqvist JK. Mental disorders in cancer suicides. J Affect Disord 1995; 36: 11-20. Doi: 10.1016/01650327(95)00047-X.

12. Bruera E, Brenneis C, Michaud M, Rafter J, Magnan A, Tennant A et al. Association between asthenia and nutritional status, lean body mass, anemia, psychological status and tumor mass in patients with advanced breast cancer. J Pain Symptom Manage 1989; 4: 59-63. Doi:10.1016/08853924(89)90023-7.

13. Lacasta MA, Calvo C, González Barón M. Psicopatología de la astenia tumoral. En: la astenia tumoral. González Barón M, Ordóñez A, editores. Madrid: Médica Paramericana; 2004. p. 91-116.

14. Mock V, Piper B, Sabbatini P, Escalante C. National Comprehensive Cancer Network fatigue practice guidelines. Oncology 2000; 14: 151-61.

15. Feliu J, Belda-Iniesta C. Tratamiento de la astenia. En: González Barón M, Ordóñez A, Feliu J, Zamora P, Espinosa E, editores. Tratado de medicina paliativa y tratamiento de soporte en el enfermo con cáncer. Panamericana; 2007. p. 443-452.

16. Bruera E, Roca E, Cedaro L, Carraro S, Chacon R. Action of oral methylprednisolone in terminal cancer patients: A prospective randomized doublé-blind study. Cancer Treatmen Rep 1985; 69: 751-4.

17. Rozans M, Dreisbach A, Lertora JJ, kahn MJ. Palliative uses of methylfenidate in patients with cancer: A review. J Clin Oncol 2002; 20: 335-9. Doi: 10.1200/ JCO.20.1.335.

18. Sanmartín Moreira J. Guías clínicas de Cuidados Paliativos. Arán; 2007. p. 121.

19. Witzig TE, Silberstein PT, Loprinzi CL, Sloan JA, Novotny PJ, Mailliard JA, et al. A phase III randomized double blind study of epoetin alfa versus placebo in anemic patients with cancer undergoing chemotherapy. J Clin Oncol 2005; 23: 2606-17. Doi: 10.1200/JCO.2004.10.020.

20. Barsevick AM, Whitmer K, Sweeney C, Nail LM. A pilot study examining energy conservation for cancer treatment-related fatigue. Cancer Nurs 2002; 25: 333-41.

21. Forester B, Kornfeld DS, Fleiss JL. Psycotherapy during radiotherapy: Effects on emotional and physical distress. Am J Psychiatry. 1985; 142: 22-7.

22. Given C, Given B, Rahbar M, et al. Effect of a cognitive behavioral intervention on reducing symptom severity during chemotherapy. J Clin Oncol. 2004; 22: 507-16. Doi: 10.1200/JCO.2004.01.241.

23. Dimeo FC. Effects of exercise on cáncerrelated fatigue. Cancer 2001; 15 (Supl. 6): 1699-707.

24. Portenoy RK, Itry LM: Cancer-related fatigue: Guidelines for evaluation and management. Oncologist 4 (1), 1999: 1-10.

25. Lacasta MA, Calvo C, González Barón M, Ordónez, A. Aspectos psicológicos de la astenia. Psicooncología 2004; 1 (2-3): 37-8.

26. Lavernia J, Guillem V. Prevención y tratamiento de la astenia. Manual SEOM de Cuidados Continuos; p.171-9.

27. Feliu J, Belda-Iniesta C. Tratamiento de la astenia. En: González Barón M, Ordóñez A, Feliu J, Zamora P, Espinosa E. Tratado de medicina paliativa y tratamiento de soporte en el enfermo con cáncer. Panamericana; 2007. p. 443-452.

28. Lacasta MA, Calvo C, González Barón M, Ordónez, A. Aspectos psicológicos de la astenia. Psicooncología 2004; 1 (2-3): 39.

29. Berger AM, Patterns of fatigue and activity and rest during adjuvant breast cáncer chemotherapy. Oncolo Nurs Forum.1998; 25: 51-62.

30. Berger AM, VonEssen S, Khun BR et al. Feasibility of a sep intervention during adjuvant breast cancer chemotherapy. Oncol Nurs Forum. 2002; 29: 1431-41. Doi: 10.1188/02.ONF.1431-1441. 